\section{Polycythemia in SARS-CoV-2 Positive Patients: An Early Screening Marker}

\section{Sir,}

The looming COVID-19 crisis imparts an imminent threat to the healthcare and economic systems of developing countries. ${ }^{1} \mathrm{As}$ of 4 th July 2020 , there are 225,281 confirmed cases and 95,570 active cases of COVID-19 in Pakistan, which has caused 4,619 deaths in the country, so far. ${ }^{2}$ Owing to the vulnerability of the healthcare system and poor implementation of lockdown policies, these numbers have taken a steep rise in the past two weeks. Moreover, due to the lack of sufficient confirmation/testing facilities and exhaustion of already existing testing laboratories, these figures, somehow underestimate the COVID-19 crisis in Pakistan.

COVID-19 updated situation in Balochistan on 4th July, reported a total, of 10,717 cases and 122 deaths. Quetta being the most populated city of the province, showed the maximum number of cases in the province and the case/fatality graph is on the rise. Only two tertiary care hospitals of the city, Fatimah Jinnah Chest and General Hospital and Sheikh Zayed Hospital, Quetta are managing symptomatic cases of COVID-19 and only one biosafety level 3 laboratory of Fatimah Jinnah Chest and General Hospital is having real-time PCR testing facility. This laboratory is receiving samples from all over the province. The limited capacity of the laboratory led to a delay in reporting, resulting pending tests.

From April2020, asymptomatic close contacts of COVID-19 positive patients or patients with mild symptoms fulfilling a case definition were tested; and upon learning of their positive status, they were shifted from quarantine facility to central diagnostic isolation centre. Fifteen positive patients were admitted into the hospital. As per the local protocols, all patients had chest X-ray and baseline laboratory tests including blood complete pictures from the same laboratory. The mean age of these patients was 29.1 years (range 25 to 45 years). All were males. Out of these patients, three were chain smokers and no one had a history of chronic pulmonary or cardiovascular pathology. A striking finding has been observed on the blood complete picture of positive COVID-19, patient; i.e. mean hemoglobin levels of $17.9 \mathrm{~g} / \mathrm{dl}$ and (Lab reference range of 13.0-17.0) and mean hematocrit of $59 \%$ (Lab reference range of $41-56 \%$ ).

Patients with COVID-19 pneumonia had abnormal coagulation parameters, according to various reports. ${ }^{3,4}$ It has been documented that a higher incidence of thrombotic events in critical
COVID-19 patients is caused by hyperfibrinogenemia. ${ }^{5} \mathrm{An}$ incidental and novel striking finding of polycythemia in COVID-19 cases, which has not been reported so far, may open new avenues for the research in the pathophysiology of disease and could also be a way forward in the screening of the disease.

There is a scarcity of evidence-based guidelines and limited resources including lack of testing facilities. A lot to be learned about specificity and sensitivity of rapid testing for SARS CoV-2. To date, across the country, the total daily capacity of RT PCR testing is 11,000 tests only. ${ }^{2}$ Blood complete picture test (a readily available and cheaper test) as a part of early screening, may help as a useful screening tool before doing RT PCR testing in otherwise healthy suspected cases.

\section{CONFLICT OF INTEREST:}

Author declared no conflict of interest.

\section{AUTHOR'S CONTRIBUTION:}

TAK: Conception, writing and editing of the manuscript.

\section{REFERENCES}

1. Wang Q, Yu C. The role of masks and respirator protection against SARS-CoV-2. Infect Control Hosp Epidemiol 2020; 41(6):746-7. doi: 10.1017/ice.2020.83.

2. Government of Pakistan. Pakistan COVID-19 Statistics. Available from: http://covid.gov.pk/stats/pakistan. (Accessed on 04/07/2020).

3. Centers for disease control and prevention (CDC). Coronavirus (COVID-19). Available from: http://www. cdc.gov/coronavirus/2019-nCoV/index.html. (Accessed on 04/07/2020).

4. Ranucci M, Ballotta A, Di Dedda U, Bayshnikova E, Dei Poli $M$, Resta $M$, et al. The procoagulant pattern of patients with COVID-19 acute respiratory distress syndrome. J Thromb Haemost 2020; 18(7):1747-51. doi: 10.1111/jth.14854.

5. Wise J. COVID-19 and thrombosis: What do we know about the risks and treatment? BMJ 2020; 369:m2058. doi: 10.1136/bmj.m2058.

Taimoor Ashraf Khan

Headquarters Ghazaband Scouts, Frontier Corps, Quetta, Balochistan, Pakistan

Correspondence to: Dr. Taimoor Ashraf Khan, Headquarters Ghazaband Scouts, Frontier Corps, Quetta, Balochistan, Pakistan

E-mail: taimoorashraf2950@gmail.com

Received: May 11, 2020; Revised: July 04, 2020; Accepted: July 14, 2020

DOI: https://doi.org/10.29271/jcpsp.2020.JCPSPCR.CR137 